\title{
Antiobesity drug therapy
}

In Reply: I thank Dr. Modarressi for these comments and agree that the impact of many glucagon-like peptide 1 (GLP-1) receptor agonists on the lowering of blood pressure is modest but significant when compared with other glucose-lowering agents and thus have exerted cardioprotective benefits.

A meta-analysis ${ }^{1}$ of 16 randomized controlled trials, including 2,417 control group participants and 3,443 patients enrolled in GLP-1 receptor agonist treatment, examined the blood pressure-lowering impact of exenatide and liraglutide.

Exenatide reduced systolic blood pressure (SBP) by a mean difference of $-5.24 \mathrm{~mm} \mathrm{Hg}$ compared with placebo $(95 \%$ confidence interval [CI] -6.88 to $-3.59, P<.001)$ and by $-3.46 \mathrm{~mm} \mathrm{Hg}$ compared with insulin glargine $(95 \% \mathrm{CI}-3.63$ to $-3.29, \mathrm{P}<.001)$. In the exenatide-treated group, diastolic blood pressure $(\mathrm{DBP})$ was reduced by $-5.91 \mathrm{~mm} \mathrm{Hg}$ compared with placebo $(95 \% \mathrm{CI}-7.53$ to $-4.28, P<.001)$ and by $-0.99 \mathrm{~mm} \mathrm{Hg}$ compared with sitagliptin $(95 \% \mathrm{CI}$ -1.12 to $-0.87, P<.001)$.

For liraglutide, SBP changes in this meta-analysis were assessed in the groups treated with $1.2 \mathrm{mg} /$ day or $1.8 \mathrm{mg} /$ day of liraglutide. In the 1.2 -mg/day group, liraglutide reduced SBP by a mean difference of $-5.60 \mathrm{~mm} \mathrm{Hg}$ compared with placebo $(95 \% \mathrm{CI}-5.84$ to $-5.36, P<.001)$ and by $-2.38 \mathrm{~mm} \mathrm{Hg}$ compared with glimepiride $(95 \% \mathrm{CI}-4.75$ to $-0.01, P=.05)$. In the 1.8-mg/day group, liraglutide also reduced SBP by $-4.49 \mathrm{~mm} \mathrm{Hg}$ compared with placebo (95\% CI -4.73 to $-4.26, P<.001)$ and by $-2.62 \mathrm{~mm} \mathrm{Hg}$ compared with glimepiride (95\% CI -2.91 to -2.33 , $P<.001)$.

In summary, treatment with the GLP-1 receptor agonists exenatide and liraglutide reduced SBP and DBP by 1 to $5 \mathrm{~mm} \mathrm{Hg}$ compared with antidiabetic drugs including insulin and glimepiride and with placebo for patients with type 2 diabetes mellitus. GLP-1 receptor agonists may offer an alternative therapy for these patients and will help provide additional cardiovascular benefits. 
Yael Mauer, MD, MPH

Department of Internal Medicine

and Geriatrics

Cleveland Clinic

Cleveland, $\mathrm{OH}$

Marcie Parker, PharmD, BCACP

Ambulatory Care Clinical Specialist

Department of Pharmacy

Cleveland Clinic

Beachwood, $\mathrm{OH}$

Sangeeta Kashyap MD

Professor of Medicine, Cleveland Clinic

Lerner College of Medicine of Case

Western Reserve University;

Associate Program Director, Endocrinology,

Diabetes, and Metabolism Fellowship

Cleveland Clinic

Cleveland, $\mathrm{OH}$

Associate Editor, Journal of Clinical

Endocrinology and Metabolism

- REFERENCES

1. Wang B, Zhong J, Lin $\mathrm{H}$, et al. Blood pressure-lowering effects

of GLP-1 receptor agonists exenatide and liraglutide: a meta-

analysis of clinical trials. Diabetes Obes Metab 2013; 15(8):737-749.

doi:10.1111/dom.12085

doi:10.3949/ccjm.88c.12002 